\title{
Lasalocid awareness and sampling in Scotland
}

\author{
Co-authors: J.W. Roxburgh ${ }^{a}$ and R.Y. Wong ${ }^{b}$ \\ ${ }^{a}$ Environmental Health, The City of Edinburgh Council, Chesser House, 500 Gorgie \\ Road, Edinburgh, EH11 3YJ, Scotland, United Kingdom. \\ Tel: 01314695770 \\ Fax: 01314695849 \\ E-mail: jeffrey.roxburgh@edinburgh.gov.uk \\ ${ }^{b}$ Environmental Health, Civil Engineering Department, University of Strathclyde, \\ John Anderson Building, 107 Rottenrow, Glasgow, G4 0NG, Scotland, United \\ Kingdom. \\ Tel: 01415483210 \\ Fax: 01415532066 \\ E-mail: raymond.wong@strath.ac.uk
}

\begin{abstract}
Lasalocid is an ionophore antibiotic extensively used as a coccidiostat in poultry production. Lasalocid should not be fed to egg-laying hens as it accumulates in the eggs, and residues have often been found in eggs. Other ionophores are toxic to humans, but the exact level of lasalocid toxicity to humans has not been established. Approximately 250 egg samples were analysed for lasalocid each year from the 10 billion eggs consumed annually in the UK. A census of the 32 Scottish Local Authority Environmental Health Departments assessed awareness of lasalocid residues in eggs, and the results indicated that awareness of lasalocid was very low and no local authorities tested for lasalocid. The example of lasalocid revealed weaknesses in the current sampling regime surrounding foods of animal origin. Conclusions are drawn that central government should raise awareness within local authorities and provide financial support on local authority sampling to achieve proper representation.

(150 words)
\end{abstract}

Keywords: Lasalocid, food sampling, eggs, coccidiostats, ionophores 


\section{Introduction}

Residues in food have been identified as cause for concern in Europe and the United Kingdom (VMD, 1995-2005). The main classes of residues include veterinary medicines, growth promoters, heavy metals and pesticides, as well as microbiological hazards (VMD, 2009). It has been reported that residues of the antibiotic lasalocid have frequently been found in eggs (Kennedy et al. 1996; Young and Nunan, 2004). Lasalocid is a member of the group of antibiotics known as the ionophores. Ionophores are extensively used to prevent coccidiosis in poultry. The ionophores exert their lethal effects on the protozoan coccidia parasites which cause coccidiosis by disrupting their osmotic balance (McKellar and Lawrence, 1996). The osmotic balance is compromised by the ionophores' powerful ability to increase the transport of cations across cell membranes. The increased influx of ions into the coccidia and the concurrent influx of extracellular water then cause the coccidia to rupture as they do not possess osmoregulatory organelles (Kart and Bilgili, 2008). The acute toxicity of lasalocid has been investigated experimentally using oral, dermal, peritoneal and subcutaneous exposure routes. These studies have shown that lasalocid is highly toxic to a wide range of species including horses, dogs, mice, rats, rabbits and chickens at very low doses via the oral route (EMEA, 2004). Adverse effects have been observed when chickens have been given feed containing even slightly more than the recommended therapeutic dose of lasalocid (Perelman et al., 1993). Lasalocid has a very narrow margin of safety, the fatal dose in chickens being only around three times the therapeutic dose (EFSA, 2007). Many cases of accidental and sometimes fatal lasalocid poisoning of horses, dogs and turkeys have been recorded and susceptibility to lasalocid toxicity varies greatly 
between animal species (EFSA, 2007; Segev et al., 2004). Extensive animal testing from previous research have shown that ingestion of lasalocid at low concentrations has powerful cardiotoxic and neurotoxic effects (Pressman and Fahim, 1983; Safran et al., 1993). Also, developmental toxic effects of lasalocid exposure have been observed in rabbits (EFSA, 2004). Drugs of lasalocid's molecular weight typically pass easily into foetal circulation and reach blood levels similar to those in the mother (Ecobichon, 1987). No information is currently available regarding lasalocid's human toxicity, but case studies on other ionophores were recorded. For example, the toxic effects of monensin and salinomycin on humans leading to serious illness and death have been confirmed and documented. A 17 year old boy developed multiple symptoms including myoglobinuria, renal failure and pulmonary congestion resulting in death 11 days after ingesting an unknown quantity of monensin (Kouyoumdjian et al., 2001). Story and Doube (2004) reported a case of serious human poisoning by salinomycin in New Zealand where a previously healthy 35 year old man was adding salinomycin granules to chicken feed. An accidental blowback occurred resulting in inhalation and swallowing of a small amount of salinomycin. Within a few minutes the man became acutely ill with symptoms of nausea, shortness of breath and dizziness. The patient's symptoms progressed to include severe muscle pain and progressive, bilateral, symmetrical leg weakness ascending to the chest and arms, closely resembling the symptoms seen in dogs poisoned with ionophores. The patient required 6 weeks of hospital treatment, and after being discharged he still had very limited exercise tolerance. In this case the patient ingested an estimated $1 \mathrm{mg} \mathrm{kg}^{-1}$ of his body weight of salinomycin. There is currently no cure or antidote available for ionophore poisoning. 
Lasalocid in animal feed is primarily used in the rations of broiler hens to protect against coccidiosis. Laying hens should not be given feed medicated with lasalocid or other ionophores as these compounds accumulate to very high concentrations in their eggs. For this reason laying hens are usually vaccinated against coccidiosis. Vaccination is usually slightly more expensive than in-feed coccidiostats but is considered financially viable for laying hens because these birds have a longer lifespan than broiler hens. However, lasalocid residues have been found in eggs when laying hens have accidentally been given feed containing lasalocid. Crosscontamination of feed in feed mills was considered to be the most likely cause when residues were found in eggs, but misuse and mistakes due to human error or mechanical equipment failures were also possible causes. Cross-contamination of feed was proved experimentally where there was carry-over of lasalocid from a medicated batch of feed to subsequent unmedicated batches in a feed mill premises which made both medicated and unmedicated feed (Kennedy et al, 1996). Eggs are a major excretion route for lasalocid (Young and Nunan, 2004) meaning that even very low levels of lasalocid in the feed can accumulate and lead to high levels of residues in the eggs. Kennedy et al., (1996) conducted an experimental feeding trial from which a concentration ratio was derived giving the equation:

\section{Concentration in Eggs $\left(\mu \mathrm{g} \mathrm{kg}^{-1}\right)=63.6 \times$ Concentration in Feed $\left(\mathrm{mg} \mathrm{kg}^{-1}\right)$}

If this equation was used as a guide, and laying hens were given medicated broiler feed containing the manufacturer's maximum recommended dose of $125 \mathrm{mg} \mathrm{kg}^{-1}$ of lasalocid, the eggs from those hens could be expected to contain almost $8,000 \mu \mathrm{g} \mathrm{kg}^{-1}$ of lasalocid (EFSA, 2007). 
Legislation has been introduced in an attempt to achieve a high level of public protection; for example, the European Regulation (EC) No. 853/2004 laid down specific hygiene rules for foods of animal origin. Under the European Council Directive 96/23/EC, Member States are required to analyse home-produced foods of animal origin for residues of veterinary medicines and environmental contaminants to ensure these foods are safe. To comply with this Directive, the U.K. Veterinary Medicines Directorate (VMD) samples eggs, among other products, as part of the statutory and non-statutory National Surveillance Programme. Approximately 10 billion eggs are consumed every year in the U.K. (BEIC, 2008), but only around 250 egg samples are analysed every year for the presence of ionophores including lasalocid. Each sample is a homogenate of 12 eggs taken from the same batch. If residues are detected in the samples, then further analysis is carried out to determine the type and concentration of the ionophore. Also, around 2 billion eggs were imported to the U.K. from European Union (EU) and non-EU countries in 2006 (BEIC, 2008), but these eggs were not subject to sampling and testing as part of the VMD's non-statutory sampling of imported produce of animal origin. In addition to the VMD statutory sampling at central government level, Environmental Health Officers from Scottish local authorities submit food samples for testing at designated laboratories every year as part of their food safety enforcement responsibilities.

Details of all the food samples submitted and the results of chemical and microbiological testing are entered on the Food Surveillance for Scotland database. Although eggs and egg products are tested under this scheme, they have not been subject to testing for the presence of ionophores such as lasalocid. Currently there are 4 Scientific Services operated under the Scottish local councils which provide chemical analysis services to all 32 local authorities in Scotland. However, these 
public analyst laboratories do not currently have the liquid chromatography/tandem mass spectrometry equipment required to analyse egg samples for the range of commonly used coccidiostats (Mackie, 2009).

In regard to the control of lasalocid in eggs, the Action Level (AL) was set by the Veterinary Medicines Directorate (VMD) at which they recorded a sample as being positive for lasalocid, and the Action Level has increased over time as illustrated in Table 1. In 2006 the European Medicines Agency introduced a Maximum Residue Limit (MRL) of $150 \mu \mathrm{g} \mathrm{kg}^{-1}$ for lasalocid in eggs although the European Food Safety Authority (EFSA) had previously stated that it was not possible to set an MRL for lasalocid (EFSA 2004). The Veterinary Medicines Directorate now use the MRL as the action level, and the setting of this MRL has effectively legalised the presence of lasalocid in eggs up to $150 \mu \mathrm{g} \mathrm{kg}^{-1}$. Figure 1 illustrated that while the sample size was relatively small, eggs have regularly been found to contain levels of lasalocid far in excess of the MRL. Despite the numerous instances of very high levels of lasalocid being found in eggs sampled by the VMD, no enforcement action has ever been implemented and no eggs have been withdrawn from sale.

The European Medicines Agency (EMEA) indicated that the Acceptable Daily Intake (ADI) for lasalocid had been set using the equation:

Acceptable Daily Intake $(\mathrm{ADI})=\frac{\text { No Observed Effect Level (NOEL) }}{\text { Uncertainty Factor }}$

The standard uncertainty factor used is 100 which was based on multiplying a factor of 10 for interspecies susceptibility by a factor of 10 for inter-individual susceptibility. EMEA stated that the safety factor which had been used to set the ADI for lasalocid was deliberately raised to 200 rather than the standard 100 in order to take into account the limited data on neurotoxicity. EMEA also confirmed that the 
ADI $(2.5 \mu \mathrm{g} / \mathrm{kg} /$ day $)$ had been derived using the lowest No Observed Effect Level (NOEL) of $0.5 \mathrm{mg} / \mathrm{kg} /$ day which was seen in a rat chronic toxicity study and in a rabbit embryo-foetal toxicity study (EMEA, 2004).

Existing data and scientific research indicated that lasalocid has been found in eggs sold in the U.K. which enter the food chain. Although records of lasalocid's human toxicity cases have not been found in the surveillance database, the aforementioned literature and research on a wide range of animal species highlighted that there are potential hazards to human health from ionophore residues in food. Also, case studies of human toxicity on other ionophores have been recorded and this indicated a health concern to consumers. While the Veterinary Medicines Directorate carries out national testing on eggs for the presence of ionophores which included lasalocid every year, the sample size is very small for proper representation of egg consumption in the U.K. Instead, much of the food safety enforcement activities which include routine sampling were undertaken at local government level. Therefore, the aim of this study was to investigate the awareness of and sampling activities for lasalocid in eggs among Scottish local authorities. The objectives were to examine the level of awareness of lasalocid among the Environmental Health Officers responsible for food safety and to undertake a statistical analysis on the existing food sampling programme on eggs in Scottish local authorities.

\section{Methods}

A survey was carried out by means of a questionnaire sent to the Environmental Health Departments of all thirty-two local authorities in Scotland. The questionnaire was designed to achieve a maximum return rate to gather data which could be 
considered representative for Scotland. The following measures were employed which have been shown to increase the return rate of postal questionnaires (Edwards et al., 2002). Initial contact by telephone to all 32 Scottish local authorities was made in order to gather and confirm accurate contact details prior to sending out the questionnaires. A specially designed questionnaire was produced and one questionnaire was sent by post to each of the Scottish local authorities, and it was addressed to the Director or Head of Environmental Health. A covering letter was also included and provided clear instruction requesting the recipient to forward the questionnaire to the Head or Leader of Food Safety group. Assurance was confirmed in the letter that names of all informants and local authorities would remain anonymous throughout the study. Follow-up telephone calls were made to the named addressees where these measures were not effective. The questionnaire was kept short consisting of only seven questions as shown in Table 2. The questions encouraged respondents to give both quantitative and qualitative answers to generate as much information as possible from a minimum number of questions, yet it could be completed in only a few minutes. None of the questions asked were of a sensitive nature.

\section{Results}

Completed questionnaires were received from all 32 local authorities in Scotland giving the desired $100 \%$ return rate. The data collected from the questionnaire provided information on two principal subjects, sampling and awareness.

The information collected included the percentage of sampling budget allocated to chemical sampling. Twenty-eight of the 32 local authorities (87.5\%) provided a 
numerical figure ranging between $4 \%-90 \%$. The data was negatively skewed with a median value of $67.5 \%$ and inter quartile range of $24.3 \%$. Also, 23 out of 28 local authorities who supplied the answers numerically have $50 \%$ or more of sampling budget spent on chemical testing. The information provided regarding the level of awareness of lasalocid in eggs among local authorities in Scotland showed that 27 of the 32 local authorities (84.4\%) had no staff members who were aware of lasalocid in eggs. Of the other 5 local authorities $(15.6 \%)$ the level of awareness was low with 2 teams stating $10 \%$ of staff were aware and the other 3 teams stating $20 \%, 25 \%$ and $33 \%$ staff awareness. The local authorities were asked whether they had any dealings with the egg or poultry industry to ascertain whether familiarity with these industries influenced their level of awareness of lasalocid. Twenty-seven of the 32 authorities (84.4\%) reported that they had dealings with the egg or poultry industries, but among these 27 authorities only 4 also had some awareness of lasalocid. Only one local authority indicated that they had carried out sampling of eggs for residues but that had not included lasalocid, and the remaining 31 authorities had not sampled eggs for lasalocid at all. All Scottish local authorities reported that they had no future plans to include lasalocid or any other residues in eggs in their sampling programmes. None of the 32 local authorities had received any enquiries from members of the public regarding lasalocid. Opinion was divided among the local authorities as to whether they perceived lasalocid residues in eggs to be a potential risk to human health. Nine authorities $(28.1 \%)$ stated that they considered lasalocid residues in eggs was a potential risk, while 7 authorities $(21.9 \%)$ stated that they did not consider it to be a risk; however, 16 authorities (50\%) stated that they did not have enough knowledge on this subject to make an informed decision. 


\section{Discussion}

\subsection{Awareness of Lasalocid in Eggs}

The VMD's statutory surveillance makes central government aware of potential food safety issues. Residues of lasalocid and other coccidiostats have been detected in eggs for many years, sometimes at very high levels. Central government delegates food safety enforcement to local authorities, but the results of the questionnaire clearly indicated that Scottish local authorities had a very low level of awareness of lasalocid residues in eggs. No authority had ever sampled eggs for lasalocid or other coccidiostat residues. Twenty-eight of the 32 Scottish Local Authorities have already reported their sampling activities of eggs and egg products as part of The Food Surveillance System (HPS 2008). Coccidiostat residue screening could be incorporated into the battery of tests currently run on these samples.

Findings from this research indicated that overall Scottish local authorities spent more of their sampling budgets on chemical than microbiological sampling. In contrast to this general trend, in recent years the percentage of samples of eggs and egg products submitted for microbiological analysis by local authorities has increased, with a consequent decrease in chemical analyses (See Figure 3). A decision has been made by local authorities to concentrate their available resources on detecting microbiological pathogens in eggs and egg products which could rapidly and severely affect the public if they were consumed. Another key factor which influenced the ratio of chemical to microbial sampling was the difference in cost of individual testings. In general, it appears that the cost of undertaking one common chemical analysis in the local authority scientific services laboratories is twice the 
amount as compared to microbiological examination (Mackie, 2009). Overall, the reduction in chemical sampling of eggs and egg products has decreased the probability of chemical contaminants being detected in these foods.

Awareness of a potential problem and its associated health risks can be seen to be important drivers for local authorities in deciding what sampling is carried out on a foodstuff. Often, a specific foodstuff will be targeted by an authority or liaison group, particularly if it has been identified as being a potential risk or is a current focus of media attention.

The Food Standards Agency also influences local authority sampling programmes by requesting them to carry out sampling of specific foodstuffs (FSA 2007), for example where there has been a food scare surrounding a particular product such as Salmonella in eggs. This is supported by the local authority returns $(22 \%)$ in which their sampling was primarily driven by issues flagged up by the FSA. There has been media coverage of the problem of lasalocid residues in eggs. This attention peaked in 2004, but awareness appears to have remained low. The VMD carries out statutory sampling: to date, the authors are unaware of any supplementary sampling having been carried out by any organization.

\subsection{Egg Sampling in Scotland}

It is not feasible and practical to examine an entire batch of eggs; this is why sampling is required so that results of analyses from the portion of the lot represented by the sample are used to draw conclusions about the whole. In order to control the level of lasalocid in eggs, a Maximum Residue Limit of $150 \mu \mathrm{g} \mathrm{kg}^{-1}$ is now imposed in an effort to limit lasalocid's effects on consumers. One of the crucial tasks is to 
determine an appropriate sample size which would be representative of the whole population and test for compliance (Barnett and O’Hagan, 1997). However, lasalocid concentration, if present, will vary in time and throughout the medium at any location. Inevitably this leads to uncertainty and sample variation. Also, the sample taken is likely to form only a fraction of the population, and any conclusion drawn about the population is subject to error. Due to the existence of sampling error, sample mean and sample proportion is merely a point estimate based on the sample.

Based on the Central Limit Theorem, the mean of the sample mean or sample proportion is approximately normally distributed regardless of the distribution of this variable under consideration as long as sample size is relatively large, and the approximation becomes better with increasing sample size (Owen and Jones, 1994). Although a larger sample size is more likely to yield a better result, in practice this is often hindered by financial and resource constraints. Ross (1987) stated that normal approximation will be reasonably good for values of $n$ (sample size) satisfying the criterion: $n p(1-p)$ greater than or equal to 10 where $p$ is the sample proportion. Based on this property, a statistical model was developed to examine the relationship between the margin of error and sample size under various sample proportions (Wong 2001).

From the data shown in Table 1, the average percentages of samples detected at or above the Action Level and the Maximum Residue Limit taken between 1998 and 2007 were used in order to obtain the smallest possible sample size but also achieve a reasonably precise estimate of the population proportion. In order to meet good normal approximation under the criterion shown by Ross (1987), the minimum sample size would be a value between 225 and 768 under $95 \%$ confidence level, as 
shown in Figure 2. The shaded area indicated possible choice of sample size $(n)$ and corresponding margin of error $(E)$ which satisfied the criterion. Therefore, where $n$ were at their minimum that satisfied good normal approximation, the $95 \%$ confidence intervals would be $4.67 \% \pm 2.8 \%$ for $n=225$ (Average AL percentage) and $1.32 \% \pm 0.8 \%$ for $\mathrm{n}=768$ (Average MRL percentage). Although these figures fulfil normal approximation, the long confidence intervals signify poor precision and the sample size $(n)$ becomes large when sample proportion $(p)$ is low.

Also, the data from Health Protection Scotland (Figure 3) illustrated the 32 Scottish local authorities' yearly sampling activities of egg and egg products between 2004 and 2007. It is shown that the total number of samples for chemical analysis dropped from 136 in 2004 to 25 in 2007. Due to the small sample size, the accuracy of these samples in representing the quality of eggs and egg products would be debatable since the margin of error would be exceedingly large.

\section{Conclusion}

The European and UK governments are committed to protecting the public health and consumer interests in relation to food. Current research indicated that ingestion of lasalocid, which has commonly been found in eggs, poses a potential risk to human health, but the results of this study confirmed that awareness of lasalocid residues among the Scottish local authorities is very low. The absence of lasalocid testing in local authority food sampling programmes is a consequence of this low level of awareness. Even if testing for lasalocid was included in the current local authority sampling regime for eggs and egg products, the small sample size, which is 
due to limited resources and manpower, would not give a true representation of the levels of lasalocid residues present in these products.

It is recommended that improved training for Environmental Health Officers in the assessment of chemical residues would help to increase awareness of lasalocid and other chemical residues in food. Also, financial support from central government is vital in tackling the issue of small sample size.

(3515 words)

\section{Acknowledgements}

The authors acknowledge the assistance of all those who took part in the survey and interviews. 


\section{References}

Barnett, V. and O'Hagan, A. 1997. Setting Environmental Standards. Chapman \& Hall, London.

British Egg Industry Council. 2008. Lion Quality Mark Information Sheet. Available: http://www.britegg.co.uk/lionquality05/startlionquality.html via the Internet.

Ecobichon, D.J. 1987. Transplacental and milk transfer of drugs: predictions from animal models. Future Health, Canadians for Health Research. Available: http://www.chrcrm.org/main via the Internet.

Edwards, P., Roberts, I., Clarke, M., DiGuiseppi, C., Pratap, S., Wentz, R., Kwan, I. 2002. Increasing response rates to postal questionnaires: systematic review. British Medical Journal 324(1183):1168-1169.

European Medicines Agency (EMEA). 2004. Committee for Veterinary Medicinal Products Lasalocid Sodium Summary Report. Available: http://www.emea.europa.eu/pdfs/vet/mrls/091204en.pdf via the Internet.

European Food Safety Authority (EFSA). 2004. Update of an opinion of the Scientific Panel on Additives and Products or Substances used in Animal Feed on the re-evaluation of coccidiostat Avatec in accordance with article $9 \mathrm{G}$ of Council directive 70/524/EEC. The EFSA Journal 77:1-45.

European Food Safety Authority (EFSA). 2007. Cross-contamination of non-target feedingstuffs by lasalocid authorised for use as a feed additive. Scientific Opinion of the Panel on Contaminants in the Food Chain. The EFSA Journal 553,1-46.

Food Standards Agency (FSA). 2007. Survey of salmonella contamination of raw shell eggs used in catering premises in the UK. Available: http://www.food.gov.uk/science/surveillance/fsisbranch2007/eggsurvey via the Internet.

Health Protection Scotland (HPS). 2008. The UK Food Surveillance System - 2007 Scotland data. Accessed from the website: http://www.hps.scot.nhs.uk.

Kart, A. and Bilgili, A. 2008. Ionophore Antibiotics: Toxicity, Mode of Action and Neurotic Aspect of Carboxylic Ionophores. Journal of Animal and Veterinary Advances 7 (6): 748-751, 2008.

Kennedy, D.G., Blanchflower, W. J., Hughes, P.J.and McCaughey, W.J. 1996. The incidence and cause of lasalocid residues in eggs in Northern Ireland. Food Additives and Contaminants 13(7): 787-794. 
Kouyoumdjian, J.A., Morita, M. da P.A., Sato, A.K. and Pissolatti, A.F. 2001. Fatal Rhabdomyolysis after Acute Sodium Monensin (Rumensin ( $)$ ) Toxicity. Case Report. Arquivos de Neuro-Psiquiatria. 59(3A).

Mackie, A. 2009. Head of Scientific Services, Edinburgh City Council. Personal communication.

McKellar, Q. and Lawrence, K. 1996. Ionophores. In Practice. September 1996: 596-598.

Owen, F. and Jones, R. 1994. Statistics ( ${ }^{\text {th }}$ Edition), Prentice Hall Publishing Limited.

Perelman, B., Pirak, M., Smith, B. 1993. Effects of the accidental feeding of lasalocid sodium to broiler breeder chickens. Veterinary Record. 132: 271-273.

Pressman, B. C. and Fahim, M. 1983. Cardiovascular toxicity of ionophores used as feed additives. Advances in Experimental Medicine and Biology. 161: 543-561.

Ross, S.M. 1987. Introduction to Probability and Statistics for Engineers and Scientists, John Wiley \& Sons, Singapore.

Safran, Z., Aizenberg, I. and Bark, H. 1993. Paralytic syndrome attributed to lasalocid residues in a commercial ration fed to dogs. Journal of the American Veterinary Association 202, 1273-1275.

Segev, G., Baneth, G., Levitin, B., Shlosberg, A. and Aroch, I. 2004. Accidental poisoning of 17 dogs with lasalocid. The Veterinary Record, August 7, 2004

Story, P. and Doube, A. 2004. A case of human poisoning by salinomycin, an agricultural antibiotic. Journal of the New Zealand Medical Association 117 (1190). Available: http://www.nzma.org.nz/journal/117-1190/799 via the Internet.

Veterinary Medicines Directorate (VMD). 1995-2005. The Veterinary Medicines Directorate Annual Report on Surveillance for Veterinary Residues. Available: http://www.vmd.gov.uk/Publications/AnnReps/AnnReps.htm via the Internet.

Veterinary Medicines Directorate (VMD). 2009. Marketing Authorisation Veterinary Information Service (MAVIS) Edition 71-July 2009. Available:

http://www.vmd.gov.uk/Publications/MAVIS/Full/mavis71.pdf via the Internet.

Wong, R.Y., Ferguson N.S. \& Clark C.F. 2001. Statistical Approach to Food Sampling. Environmental Health Risk, WIT Press, Southampton, 227-234.

Young, R. and Nunan, C. 2004. Too hard to crack? Eggs with drug residues. Report Four in The use and misuse of antibiotics in UK agriculture series. Soil Association. 